\title{
Born-again AGB stars: Starting point of the H-deficient post-AGB evolutionary sequence?
}

\author{
K. Werner, S. Dreizler, T. Rauch
}

Institut für Astronomie und Astrophysik, Univ. Tübingen, Germany

L. Koesterke

Institut für Physik, Universität Potsdam, Germany

U. Heber

Sternwarte Bamberg, Universität Erlangen-Nürnberg, Germany

\begin{abstract}
We discuss the origin of hot hydrogen-deficient post-AGB stars. Evidence is growing that the "born-again AGB star scenario" as a consequence of a late He shell flash cannot explain the diverse observations. Analyses of the stars and their associated planetary nebulae suggest that the H-deficiency is not formed during the second post-AGB phase but already while the star is still on the AGB. There are even hints that the H-deficiency can occur before the first departure from the AGB.
\end{abstract}

\section{Introduction}

Hot post-AGB stars $\left(T_{\text {eff }}>20000 \mathrm{~K}\right)$ can be grouped into either H-deficient or H"normal" objects. The evolution of the latter group is in principle understood, so we concentrate here on the problems with H-deficient objects. Spectroscopic analyses during the recent years have firmly established an evolutionary sequence formed by the Wolf-Rayet central stars of planetary nebulae (CSPN) and the PG 1159 stars. This is suggested by their location in the $g$ - $T_{\text {eff-plane (Fig. } 1)}$ as well as the similarity of their chemical abundance patterns. The Wolf-Rayet CSPN, dominated by $\mathrm{He}, \mathrm{C}$, and $\mathrm{O}$ emission lines, are subdivided into late and early spectral types, [WCL] and [WCE]. The link between the [WCE] and PG 1159 stars is marked by a few [WC]-PG 1159 transition objects which show a mixed emission/absorption line spectrum. The PG 1159 stars display essentially absorption line spectra. While [WC] stars are known for a long time, the PG 1159 stars have been established as a spectral class more recently (Wesemael et al. 1985). We know 31 PG 1159 stars and every other is a CSPN. Note that the so-called "OVI" classification of CSPN is no longer used because objects having very different spectra were subsumed under it. All former "OVI" stars are now [WCE] stars of different sub-classes according to the classification scheme of Méndez \& Niemela (1982). An exception is the well known CSPN NGC 246, also a former "OVI" object, which is now classified as PG 1159. 


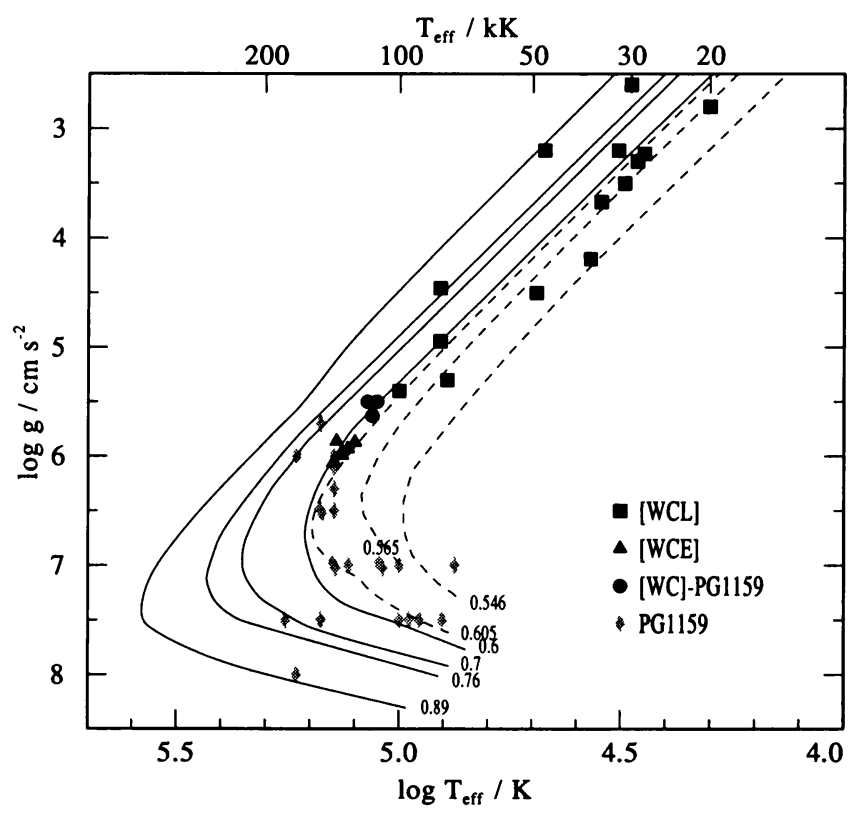

Figure 1. Hydrogen-deficient post-AGB stars in the $g-T_{\text {eff }}$-plane. We identify Wolf-Rayet CSPN (Hamann 1997), PG 1159 stars as well as [WC]-PG 1159 transition objects. Evolutionary tracks are from Schönberner (1983, dashed lines) and Wood \& Faulkner (1986) (labels: mass in $\left.\mathrm{M}_{\odot}\right)$. They are only shown for a rough comparison, because they predict normal, H-rich surface abundances. $T_{\text {eff }}$ for the [WC] stars is defined related to the stellar radius at $\tau_{\text {Ross }}=20$.

For detailed spectroscopic results on the [WC] stars we refer to a review by Hamann (1997). An overview about results on PG 1159 stars is found in Werner et al. (1996), and latest results based on HST spectroscopy were published by Dreizler \& Heber (1998).

\section{Origin of H-deficient post-AGB stars}

The typical chemical composition of PG 1159 atmospheres is displayed by the prototype PG 1159-035 itself. We found He:C:O=33:50:17 (mass fractions) while $\mathrm{H}$ is not detectable. A comparison of this abundance pattern with the interior structure of post-AGB stellar models suggests that the PG 1159 stars must have lost their entire H-rich envelope $\left(\approx 10^{-4} \mathrm{M}_{\odot}\right)$ and also most of their He-rich intershell layer $\left(\approx 10^{-2} \mathrm{M}_{\odot}\right)$ because the high $\mathrm{C}$ and $\mathrm{O}$ abundances are found only at its bottom. With the first analyses of PG 1159 stars (Werner et al. 1991), we suggested that their H-deficiency is a consequence of a late He shell flash. Originally, Iben (1984) introduced this scenario in order to explain the discovery of H-deficient zones in the inner parts of the PNe Abell 30 and Abell 78 (whose nuclei are today classified as [WC]-PG 1159 transition objects). In this scenario He shell burning re-ignites when the post-AGB star is already on the WD cooling 
track. As a consequence, the star moves back to the AGB ("born-again" AGB star). The star suffers another superwind phase and during its second descent from the AGB the star looses its remaining $H$ envelope by a fast wind.

There are several problems with this scenario, which suggest that the $\mathrm{H}$ deficiency is not only formed during the second post-AGB evolution, but already during the second AGB phase. In spite of the rapid evolutionary rate, the existence of cool (i.e. young) [WC] stars requires the complete ejection of the $\mathrm{H}$-rich envelope on the AGB. This is also corroborated by the fact that the ejection of H-deficient material in the PNe Abell 30 and Abell 78 occurs with low expansion velocities ( $<100 \mathrm{~km} \mathrm{~s}^{-1}$, Borkowski et al. 1995), which means that already the AGB precursor of the CSPN must have been H-deficient.

The born-again scenario is faced with another problem, which is related to the expansion ages of the nebulae of [WC] stars. Their PNe should be systematically older than those of the CSPN with normal surface chemistry, however, this is not the case (Górny \& Stasińska 1995). This finding suggests that the H-deficiency occurs possibly at the first departure from the AGB, i.e. without invoking the born-again scenario at all. What then is the origin of the H-deficiency? One possibility is close binary evolution during which the stellar envelope could be stripped off by interaction with the companion star. However, for [WC] stars binarity is extremely difficult to detect directly from radial velocity or photometry. For PG 1159 stars the binary frequency does not appear to be unusually high (Napiwotzki 1998). Bipolarity of PNe has frequently been invoked as a tracer of binarity. Here we face a puzzling result: while the frequency of bipolar PNe around [WC] stars appears to be "normal" (Górny \& Stasińska 1995), the fraction of PG 1159 stars with a bipolar PN appears to be high (3/4, Napiwotzki et al. 1996). The latter result, however, is limited by small number statistics and inhomogeneous observational material.

A further problem is the high photospheric $\mathrm{O}$ abundance found in many PG 1159 and [WC] stars, which approaches almost $20 \%$ by mass. As already mentioned above, this means that not only the $\mathrm{H}$ envelope must be removed from the star, but also the 100 times more massive He envelope. This conflicts even more severely with the existence of the young [WCL] stars: removal of the He-rich intershell during the post-AGB evolution can be enforced in theoretical calculations by high mass-loss rates, but envelope removal is not completed until the star is very hot $\left(T_{\text {eff }}>100000 \mathrm{~K}\right)$, because the main effect of mass-loss is to increase the evolutionary rate (Schönberner \& Blöcker 1992). However, new evolution calculations show that this problem is probably much less severe. These calculations, which include a hydrodynamically based overshoot prescription (Herwig etal. 1997), provide a very high $\mathrm{O}$ abundance throughout the He-rich intershell, and not only at its bottom as suggested by earlier computations. Though the problem of envelope removal seems less serious now, it is still unsolved. Nevertheless, we have to consider the exceptional case of $\mathrm{H} 1504+65$, a PG 1159 star which is completely devoid of $\mathrm{H}$ and He, i.e. its atmosphere is composed only of $\mathrm{C}$ and $\mathrm{O}$, by equal parts (Werner 1991; Kruk \& Werner 1998). Obviously this naked $\mathrm{C} / \mathrm{O}$ stellar core must have lost also its massive He-rich intershell.

Further evidence that the H-deficiency is formed on the AGB or at least very early on the post-AGB track comes from the analysis of ISO spectroscopy 
of two [WCL] CSPN (Waters et al. 1998). C-rich dust (PAHs) in the inner part of the PN and crystalline (O-rich) silicates in the outer part suggest that the chemical composition of both stars changed to a $\mathrm{C}$-rich mixture very recently, i.e. less than 1000 years ago. The $\mathrm{H}$-rich nature of the $\mathrm{C}$-rich dust suggests that still some $H$ was left on the star, which is in accordance with the identification of $\mathrm{H}$ in one of these CSPN (Leuenhagen \& Hamann 1998).

The detection of residual H in some [WC] and PG 1159 stars (Dreizler et al. 1997) as well as the detection of $\mathrm{N}$ in a few cases suggest that mixing and incomplete burning of the $\mathrm{H}$ envelope take place in these stars. According to evolutionary calculations this can only occur after a very late He shell flash (i.e. on the WD cooling track, where $\mathrm{H}$ shell burning is extinct) and not on or soon after the AGB phase (where $\mathrm{H}$ shell burning is active; Iben \& MacDonald 1995). This is in clear contradiction with the PN observations of $\mathrm{H}$-deficient gas and dust around the stars (see above).

To conclude, the true origin for H-deficiency in post-AGB stars is not known. Further progress can be expected from evolutionary calculations which must include both overshoot as described above (Herwig et al. 1997) and time-dependent mixing as presented in an exploratory study by Iben \& MacDonald (1995).

\section{Another H-deficient post-AGB sequence}

A few hot H-deficient post-AGB stars cannot be classified as PG 1159 stars, because their absorption line spectra are exhibiting almost exclusively He lines and, if at all, only weak $\mathrm{C}$ or other metal lines. This small group of stars comprises four objects, two of which have an associated PN. The stars are classified as "O(He)" according to the scheme of Méndez et al. (1986) and the prototype is the CSPN K1-27. Recent analyses by Rauch et al. $(1994,1996,1998)$ confirm that only trace amounts of metals are present in the He-dominated atmospheres but, interestingly, two of them have a considerable fraction of $\mathrm{H}$ left $(10 \%$ and $30 \%$, by number). They are very hot $\left(T_{\text {eff }}=100000-140000 \mathrm{~K}\right)$ and have relatively low surface gravities $(\log g=5.5-6.5)$, hence, their location in the $g-T_{\text {eff }}$-plane is among the PG 1159 and [WCE] stars. Rauch et al. suggest that the $\mathrm{O}(\mathrm{He})$ stars are the successors of He-rich sdO stars, which are on post-AGB evolutionary tracks (Husfeld et al. 1989). The variety of abundance patterns among PG 1159 stars and [WC] stars is rather diverse and the existence of $\mathrm{O}(\mathrm{He})$ stars makes an explanation for the origin of $\mathrm{H}$-deficient stars even more difficult. We can at present only speculate that, among other details, the phase in the thermal pulse cycle at the moment of AGB departure plays an important role.

\section{Mass-loss from H-deficient post-AGB stars}

From the above discussions it is clear that the knowledge about mass-loss from [WC] and PG 1159 stars is essential to our understanding of the H-deficient post-AGB stars. Mass-loss rates from [WC] stars can be determined by the analyses of the optical emission line spectra. Good spectral resolution is required in order to eliminate the contribution from nebular emission lines to the photospheric features. As a result, high mass-loss rates are found, ranging between $10^{-5}-10^{-6.5} \mathrm{M}_{\odot} / \mathrm{yr}$, with rates decreasing along the post-AGB evolu- 
tion. The mass-loss rate of two [WC]-PG 1159 transition objects amounts to $10^{-7.5} \mathrm{M}_{\odot} / \mathrm{yr}$ (Werner \& Koesterke 1992; Hamann 1997). For the PG 1159 stars one expects even smaller rates. As a consequence, the optical spectrum is formed in the hydrostatic layers of the photosphere and only the strong UV resonance lines of CIV and O VI can be used to study the properties of the weak winds. We have analyzed IUE, HST and ORFEUS spectra of four low gravity PG 1159 stars, i.e. immediate successors of [WCE] stars, and found $\dot{M}=10^{-7}-10^{-8} \mathrm{M}_{\odot} / \mathrm{yr}$ (Koesterke et al. 1998; Koesterke \& Werner 1998). Comparison with theoretical studies (Pauldrach et al. 1988) suggests that the winds are radiation driven. For the [WC] stars the highly stratified ionization structure of metals in our photospheric models lends further support to the hypothesis that the strong [WC] winds are radiation driven, too. This might explain why the supposed progenitors of the $\mathrm{O}(\mathrm{He})$ stars, the He-rich and metal poor sdO-stars, do have "normal" mass-loss rates (only observable in the UV spectra) in contrast to the C- and O-rich [WC] stars which are found in the same place of the $g-T_{\text {eff }}-$ plane.

\section{Occurrence of super-hot winds at the end of pre-WD evolution}

The bifurcation of post-AGB evolution into H-deficient and H-normal channels seems to continue into the WD evolution, dividing the cooling sequence into nonDA and DA white dwarfs. It is natural to assume that the hottest He-rich white dwarfs (spectral type DO) are descendants of the PG 1159 stars, however, some $\mathrm{H}$-deficient post-AGB stars will become DA white dwarfs, namely those who have retained at least some $\mathrm{H}$ in their envelope. The transition of post-AGB stars into the hot WD phase is encompassed by a spectacular phenomenon, which has been discovered only recently. We have found that a large fraction (50\%) of the hot DO white dwarfs shows signatures of a super-hot wind (Werner et al. 1995; Dreizler et al. 1995) in the optical spectrum. We have discovered high ionization absorption lines of the CNO elements (e.g. C VI, N VII, O VIII, and even NeX). The high excitation potentials involved require temperatures approaching almost one million $\mathrm{K}$ and the triangular shaped line profiles suggest their formation in a rapidly accelerating wind from the $W D$, reaching a terminal speed of the order of $10000 \mathrm{~km} \mathrm{~s}^{-1}$. Preliminary model calculations suggest mass-loss rates between $10^{-7}$ and $10^{-10} \mathrm{M}_{\odot} / \mathrm{yr}$. However, at present these radiative equilibrium models are crude at best, as they simply assume a very high $T_{\text {eff }}$ in order to produce the high ionization stages. Since the post-AGB age is of the order of $10^{5}-10^{6} \mathrm{yr}$, consequences of the stellar wind on evolutionary times scales and chemical surface composition are possible, if the mass-loss rates are in fact as high as estimated right now.

Finally we emphasize that this wind phenomenon is not restricted to DO white dwarfs. We have also detected one DAO white dwarf with similar high ionization features (Dreizler et al. 1995). This is the only case discovered among $\mathrm{DA} / \mathrm{DAO}$ white dwarfs so far, and the puzzle arises why there is such a strong preference among DOs to show this phenomenon.

Acknowledgments. HST and ORFEUS data analysis is supported by the Deutsches Zentrum für Luft- und Raumfahrt (DLR) under grants 50 OR 9705 and 50 QV 97054 (in Tübingen) and 50 OR 96029 and 50 QV 97026 (in Bamberg). 


\section{References}

Borkowski K.J., Harrington J.P., Tsvetanov Z.I., 1995, ApJ 449, L143

Dreizler S., Heber U., 1998, A\&A 334, 618

Dreizler S., Heber U., Napiwotzki R., Hagen H.-J., 1995, A\&A 303, L53

Dreizler S., Werner K., Heber U., 1997, IAU Symp. 180, Kluwer, p. 103

Górny S.K., Stasińska G., 1995, A\&A 303, 893

Hamann W.-R., 1997, IAU Symp. 180, Kluwer, p. 91

Herwig F., Blöcker T., Schönberner D., El Eid M., 1997, A\&A 324, L81

Husfeld D., Butler K., Heber U., Drilling J.S., 1989, A\&A 222, 150

Iben I., Jr., 1984, ApJ 277, 333

Iben I., Jr., MacDonald J., 1995, in White Dwarfs, eds. D. Koester and K.

Werner, Springer, Lecture Notes in Physics 443, p. 48

Koesterke L., Werner K., 1998, ApJ 500, L55

Koesterke L., Dreizler S., Rauch T., 1998, A\&A 320, 1041

Kruk J.W., Werner K., 1998, ApJ 502, 858

Leuenhagen U., Hamann W.-R., 1998, A\&A 330, 265

Méndez R.H., Niemela V.S., 1982, IAU Symp. 99, p. 457

Méndez R.H., Miguel C.H., Heber U., Kudritzki R.P., 1986, IAU Coll. 87, Reidel, Dordrecht, p. 323

Napiwotzki R., 1998, in The Third Conference on Faint Blue Stars, eds. A.G.D. Philip, J. Liebert and R.A. Saffer, in press

Napiwotzki R., Haas, S., Schönberner, D., 1996, in Hydrogen-Deficient Stars, eds. U. Heber and C.S. Jeffery, ASP Conference Series 96, p. 213

Pauldrach A., Puls J., Kudritzki R.P., Méndez R.H., Heap S.R., 1988, A\&A 207,123

Rauch T., Köppen J., Werner K., 1994, A\&A 286, 543

Rauch T., Köppen J., Werner K., 1996, A\&A 310, 613

Rauch T., Dreizler S., Wolff B., 1998, A\&A 338, 651

Schönberner D., 1983, ApJ 272, 708

Schönberner D., Blöcker T., 1992, in The Atmospheres of Early-Type Stars, eds. C.S. Jeffery and U. Heber, Springer, Lecture Notes in Physics 401, p. 305

Waters L.B.F.M., Beintema D.A., Zijlstra A.A., et al., 1998, A\&A 331, L61

Werner K., 1991, A\&A 251, 147

Werner K., Koesterke L., 1992, in The Atmospheres of Early-Type Stars, eds.

C.S. Jeffery and U. Heber, Springer, Lecture Notes in Physics 401, p. 288

Werner K., Heber U., Hunger K., 1991, A\&A 244, 437

Werner K., Dreizler S., Heber U., Rauch T., Wisotzki L., Hagen H.-J., 1995, A\&A 293, L75

Werner K., Dreizler S., Heber U., Rauch T., 1996, in Hydrogen-Deficient Stars, eds. U.Heber and C.S.Jeffery, ASP Conference Series 96, p. 267

Wesemael F., Green R.F., Liebert J., 1985, ApJS 58, 379

Wood P.R., Faulkner D.J., 1986, ApJ 307, 659 